\title{
Vanillic acid alleviates lipopolysaccharides-induced endoplasmic reticulum stress and inflammation in human lung fibroblasts by 'inhibiting MAPK and NF-KB pathways
}

\author{
Jihua Zhao, Yao Yang*
}

Neonatal.Child Critical Child Health Care Division, The Central Hospital of Enshi Tujia and Miao Autonomous Prefecture, Enshi Tujia and Miao Autonomous Prefecture, Hubei Province, China

"Corresponding Author: Yao Yang, Neonatal Child Critical.Child Health Care Division, The Central Hospital of Enshi Tujia and Miao Autonomous Prefecture, No. 88, Middle Section, Jinlong Avenue, Enshi Tujia and Miao Autonomous Prefecture, Hubei Province, 445000, China. Email: yang003215@126.com

Received: 29 November 2021; Accepted: 15 December 2021; Published: 21 January 2022 (c) 2022 Codon Publications

\begin{abstract}
Persistent endoplasmic reticulum stress promotes aberrant inflammation and induces cell death, and inflammation is implicated in the pathogenesis of pneumonia. Vanillic acid exerts pharmacological activities, such as anti-inflammatory, antimicrobial, and antioxidant effects. However, the role of vanillic acid in pneumonia has not been elucidated yet. Human lung fibroblasts (WI-38 and MRC-5) were incubated with different concentrations of lipopolysaccharides to mimic the cell model of pneumonia. Lipopolysaccharides-treated lung fibroblasts were then incubated with different concentrations of vanillic acid. Cell viability and apoptosis were detected by MTT assay and flow cytometry, respectively. Quantitative real-time polymerase chain reaction and enzyme-linked-immunosorbent serologic assay were used to measure the levels of inflammatory factors. Western blot assay was used to detect endoplasmic reticulum stress and downstream pathway. Lipopolysaccharides induced decrease of cell viability in WI-38 and MRC-5 whereas vanillic acid increased cell viability of lipopolysaccharides-treated lung fibroblasts. Lipopolysaccharides-induced increase of cell apoptosis in lung fibroblasts was suppressed by vanillic acid through up-regulation of $B C L 2$, and down-regulation of $B C L 2$ associated $\mathrm{X}(B A X)$ and cleaved caspase-3. Vanillic acid reduced levels of tumor necrosis factor- $\alpha$ (TNF- $\alpha$ ), Interleukin 6 (IL-6), and IL-1 $\beta$ in lipopolysaccharides-treated lung fibroblasts. Protein expression of glucose-regulated protein 78 (GRP78), X-box binding protein 1 (XBP-1), activating transcription factor-6 (ATF-6), ATF-4, and C/EBP homologous protein (CHOP) in lung fibroblasts were up-regulated by lipopolysaccharides while reduced by vanillic acid. Vanillic acid attenuated lipopolysaccharides-induced decrease of I $\mathrm{B} \alpha$ and increase of $\mathrm{p}-\mathrm{I} \kappa \mathrm{B} \alpha$, p-p65, p-ERK, and p-JNK in fibroblasts. Vanillic acid exerted anti-inflammatory effect against lipopolysaccharides-induced human lung fibroblasts by inhibiting mitogen-activated protein kinase and nuclear factor kappa B pathways.
\end{abstract}

Keywords: vanillic acid, lipopolysaccharides, inflammation, endoplasmic reticulum stress, human lung fibroblasts, MAPK, NF- $\kappa B$, pneumonia

\section{Introduction}

Pneumonia is one of the most common respiratory diseases in children and is the major cause of death in children aged less than 5 years (Hur, 2020). Almost one-third of neonatal deaths are caused by pneumonia, and the incidence of pneumonia in developing countries is on the rise (Marangu and Zar, 2019). Therapeutic strategies, including antibiotics, nebulizer therapy, and oxygen inhalation, are widely used for the treatment of pediatric pneumonia (Dassner et al., 
2017; Müller-Redetzky et al., 2015). However, resistance to antibiotics weakens the therapeutic efficiency (MüllerRedetzky et al., 2015). Therefore, novel strategies for the treatment of pneumonia are required urgently.

Bacterial or viral infections induce inflammatory response in the lungs, leading to pneumonia (Mizgerd, 2018). The severity of pneumonia is strongly correlated with the degree of inflammation (Mizgerd, 2018). Anti-inflammatory therapy has been regarded as the potential strategy for preventing pneumonia (Seki et al., 2010). In addition, environmental stimulus induces loss of endoplasmic reticulum homeostasis and exacerbate inflammatory process in the lungs, thus resulting in development of pneumonia (Köseler et al., 2020). Suppression of endoplasmic reticulum stress reduced lipopolysaccharides-induced lung inflammation (Kim et al., 2013) and repressed lung cell apoptosis and autophagy (Huang et al., 2020). Therefore, inhibition of endoplasmic reticulum stress-induced inflammation could be a promising treatment for pneumonia.

Vanillic acid is a phenolic compound derived from dietary fruit and vegetation, and exerts a variety of pharmacological activities, such as anti-inflammatory, antimicrobial, antioxidant, and neuroprotective effects (Ullah et al., 2020). For instance, vanillic acid improved cognitive impairment in mice and suppressed $A \beta_{1-42}$-induced oxidative stress, thus exerting neuroprotective effect against progressive neurodegenerative diseases (Amin et al., 2017). Vanillic acid also promoted myelination and inhibited inflammation to ameliorate neurodegenerative diseases (Siddiqui et al., 2019). Streptozotocin-induced inflammation and oxidative stress were also attenuated by vanillic acid (Ji et al., 2020). Vanillic acid has been indicated to reduce the release of inflammatory cytokines in bronchoalveolar lavage fluid, and attenuated ovalbumin-induced asthma (Bai et al., 2019). However, the role of vanillic acid in pediatric pneumonia has not been reported yet.

In the present study, the effects of vanillic acid on cell viability, apoptosis, inflammation, and endoplasmic reticulum stress of lipopolysaccharides-treated human lung fibroblasts were investigated. The underlying mechanism of anti-inflammatory effect of vanillic acid against lipopolysaccharides-treated human lung fibroblasts could provide potential strategy for pediatric pneumonia.

\section{Materials and methods}

\section{Effect of vanillic acid on cell viability and lipopolysaccharides-induced apoptosis}

Human lung fibroblasts (WI-38 and MRC-5) were acquired from American Type Culture Collection
(Manassas, VA, USA), and cultured in Dulbecco's modified Eagle's medium (Invitrogen, Carlsbad, CA, USA) supplemented with $10 \%$ fetal bovine serum and penicillin-streptomycin (Invitrogen). Cells were incubated with 1-, 2-, 5-, or 10- $\mu \mathrm{g} / \mathrm{mL}$ lipopolysaccharides (Sigma-Aldrich, St Louis, MO, USA) for $12 \mathrm{~h}$ to induce inflammation. Cells were also incubated with 5-, 10-, 20-, or $40-\mu \mathrm{g} / \mathrm{mL}$ vanillic acid (Sigma-Aldrich) for $12 \mathrm{~h}$ to investigate cytotoxicity. Cells were cotreated with $10-\mu \mathrm{g} /$ $\mathrm{mL}$ lipopolysaccharides and 5-, 10-, or $20-\mu \mathrm{g} / \mathrm{mL}$ vanillic acid to investigate the protective effect of vanillic acid.

WI-38 and MRC-5 were seeded into 96-well plates, and treated with lipopolysaccharides or vanillic acid. MTT solution $(5 \mathrm{mg} / \mathrm{mL}, 10 \mu \mathrm{L})$ (Sigma-Aldrich) was added into each well and incubated at $37^{\circ} \mathrm{C}$ for $4 \mathrm{~h}$. Dimethyl sulfoxide was then added and absorbance at $450 \mathrm{~nm}$ was measured by microplate reader (Bio-Rad, Hercules, CA, USA). For flow cytometry, WI-38 and MRC-5 with lipopolysaccharides or vanillic acid treatment were harvested and resuspended in binding buffer of Annexin V fluorescein isothiocyanate (FITC)/propidium iodide (PI) staining assay (Invitrogen). Cells were then stained with $5 \mu \mathrm{L}$ of PI and $5 \mu \mathrm{L}$ of Annexin $\mathrm{V}$ (Invitrogen). The apoptotic ratio was analyzed by fluorescence-activated cell sorting (FACS) flow cytometer (Life Technologies, Darmstadt, Germany).

\section{Enzyme-linked-immunosorbent serologic assay (ELISA) and quantitative real-time polymerase chain reaction (qRT-PCR)}

The cultured medium of WI-38 and MRC-5 was collected, and the protein concentration was measured by BCA kit (Applygen, Beijing, China). Levels of tumor necrosis factor- $\alpha$ (TNF- $\alpha$ ), Interleukin 6 (IL-6), and IL-1 $\beta$ were determined using ELISA kits (ExCell Biology Inc., Shanghai, China). For qRT-PCR analysis, WI-38 and MRC-5 were lysed in TRIzol kit (Invitrogen), and the isolated RNAs were synthesized into complementary DNAs (cDNAs) by Multiscribe ${ }^{\mathrm{TM}}$ reverse transcription kit (Applied Biosystems, CA, USA). PreTaq II kit (Takara, Dalian, Liaoning, China) was used for the qRT-PCR analysis with the primers given in Table 1 . The messenger RNA (mRNA) expression was normalized to glyceraldehyde 3-phosphate dehydrogenase (GAPDH) through $2^{-\Delta \Delta \mathrm{Ct}}$ method.

\section{Western blot assay}

WI-38 and MRC-5 were lysed in radioimmunoprecipitation assay (RIPA) buffer (Beyotime, Beijing, China), and the protein samples were collected and separated by $10 \%$ sodium dodecyl sulfate-polyacrylamide gel electrophoresis (SDS-PAGE). Samples were transferred on 
Table 1. Primer sequences.

\begin{tabular}{lll} 
Gene & Forward & Reverse \\
\hline TNF- $\alpha$ & 5'-AGCCCATGTTGTAGCAAACC-3' & 5'-GCTGGTTATCTCTCAGCTCCA-3' \\
IL-6 & 5'-GGTGAGTGGCTGTCTGTGTG-3' & 5'-TTCGGTCCAGTTGCCTTCT-3' \\
IL-1 $\beta$ & 5'-GTGGCAATGAGGATGACTTG-3' & 5'-TGGTGGTCGGAGATTCGTA -3' \\
GAPDH & 5'-TCAACGACCACTTTGTCAAGCAGAGT-3' & 5'-GCTGGTGGTCCAGGGGTCTTACT-3' \\
\hline
\end{tabular}

nitrocellulose membranes, and the membranes were blocked in 5\% bicinchoninic acid (BCA). Following overnight incubation with specific antibodies: anti- BCL2 associated $\mathrm{X}(B A X)$ and anti-BCL2 (1:1,500, Abcam, Cambridge, UK), anti-GRP-78 and anti-XBP-1 (1:2,000, Abcam), anti-activating transcription factor-6 (anti-ATF-6) and anti-ATF-4 (1:2,500, Abcam), anti-C/EBP homologous protein (CHOP) and anti- $\beta$-actin $(1: 3,000$, Abcam), antipro caspase- 3 and anti-cleaved caspase 3 (1:3,000, Abcam), anti-JNK and anti-p-JNK (1:3,500, Abcam), anti-ERK and anti-p-ERK (1:4,000, Abcam), anti-p65 and anti-p-p65

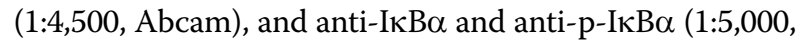
Abcam), the membranes were washed and probed with horseradish peroxidase-conjugated secondary antibody (1:5,000, Abcam). The immunoreactivities were visualized using enhanced chemiluminescence (Sigma-Aldrich) followed by incubation with tetramethylbenzidine.

\section{Statistical analysis}

Complete data with at least triple replicates were expressed as mean \pm standard error of mean (SEM), and analyzed by Student's $t$-test or one-way analysis of variance under SPSS software. $P<0.05$ was considered statistically significant.

\section{Results}

\section{Vanillic acid suppressed cell apoptosis of lipopolysaccharides-treated human lung fibroblasts}

In order to induce cell model of pneumonia, human lung fibroblasts (WI-38 and MRC-5) were incubated with lipopolysaccharides. Incubation with lipopolysaccharides reduced cell viabilities of WI-38 and MRC-5 (Figure 1A). Lipopolysaccharides below less than $2 \mu \mathrm{g} /$ $\mathrm{mL}$ showed demonstrated no significant effect on the cell viabilities (Figure $1 \mathrm{~A}$ ), and thebut $10-\mu \mathrm{g} / \mathrm{mL}$ lipopolysaccharides reduced almost $50 \%$ of cell viabilities in WI-38 and MRC-5 (Figure 1A). In order to investigate cytotoxicity of vanillic acid on human lung fibroblasts, WI-38 and MRC-5 were incubated with 5-, 10-, 20-, or 40- $\mu \mathrm{g} /$ $\mathrm{mL}$ vanillic acid. Vanillic acid less thanbelow $20 \mu \mathrm{g} / \mathrm{mL}$ depictedshowed no significant effect on the cell viabilities of WI-38 and MRC-5 (Figure 1B). Therefore, to investigate the protective effect of vanillic acid, cells were cotreated with $10-\mu \mathrm{g} / \mathrm{mL}$ lipopolysaccharides and 5-, 10-, or 20- $\mu \mathrm{g} /$ $\mathrm{mL}$ vanillic acid. Incubation with vanillic acid enhanced cell viabilities of lipopolysaccharides-treated WI-38 and MRC-5 in a dosage-dependent way manner (Figure 1C), and reduced the cell apoptosis (Figure 1D). Moreover, vanillic acid treatment decreased protein expression of BAX, while increased BCL2 and cleaved caspase 3 in lipopolysaccharides-treated WI-38 and MRC-5 (Figure 1E), suggesting anti-apoptotic effect of vanillic acid against lipopolysaccharides-treated human lung fibroblasts.

\section{Vanillic acid suppressed inflammation of lipopolysaccharides-treated human lung fibroblasts}

Expression levels of TNF- $\alpha$, IL-6, and IL- $1 \beta$ were upregulated in WI-38 and MRC-5 post-lipopolysaccharides incubation (Figures $2 \mathrm{~A}$ and $\mathrm{B}$ ). However, vanillic acid treatment reduced the levels of TNF- $\alpha$, IL- 6 , and IL-1 $\beta$ in lipopolysaccharides-treated WI-38 and MRC-5 in a dosage-dependent manner (Figures $2 \mathrm{~A}$ and $\mathrm{B}$ ), demonstrating the anti-inflammatory effect of vanillic acid against lipopolysaccharides-induced inflammation in human lung fibroblasts.

\section{Vanillic acid suppressed endoplasmic reticulum stress of lipopolysaccharides-treated human lung fibroblasts}

Protein expressions of GRP-78, XBP-1, ATF-6, ATF4, and CHOP were up-regulated in WI-38 and MRC-5 post-lipopolysaccharides incubation (Figure 3). However, vanillic acid treatment reduced the expression of GRP-78, XBP-1, ATF-6, ATF-4, and CHOP in lipopolysaccharidestreated WI-38 and MRC-5 in a dosage-dependent manner (Figure 3), demonstrating anti-endoplasmic reticulum stress effect of vanillic acid against lipopolysaccharidestreated human lung fibroblasts.

\section{Vanillic acid suppressed activation of mitogen-activated protein kinase (MAPK) and nuclear factor kappa B (NF-kB) pathways}

Although protein expressions of JNK and ERK in WI-38 and MRC-5 were not affected by lipopolysaccharides 
$(\mathrm{A})^{-}$
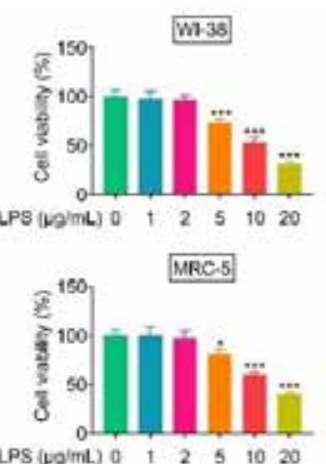

(B)
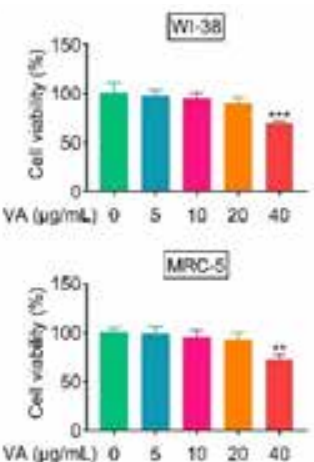

(C)

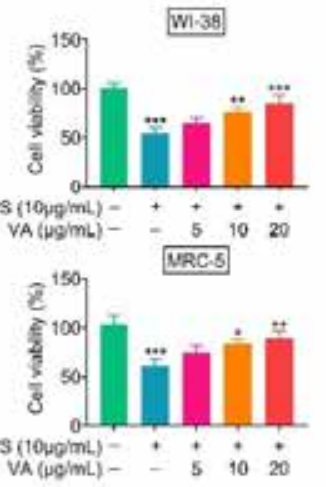

(D)

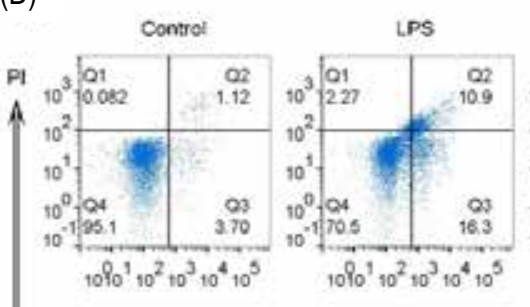

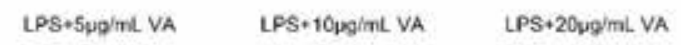
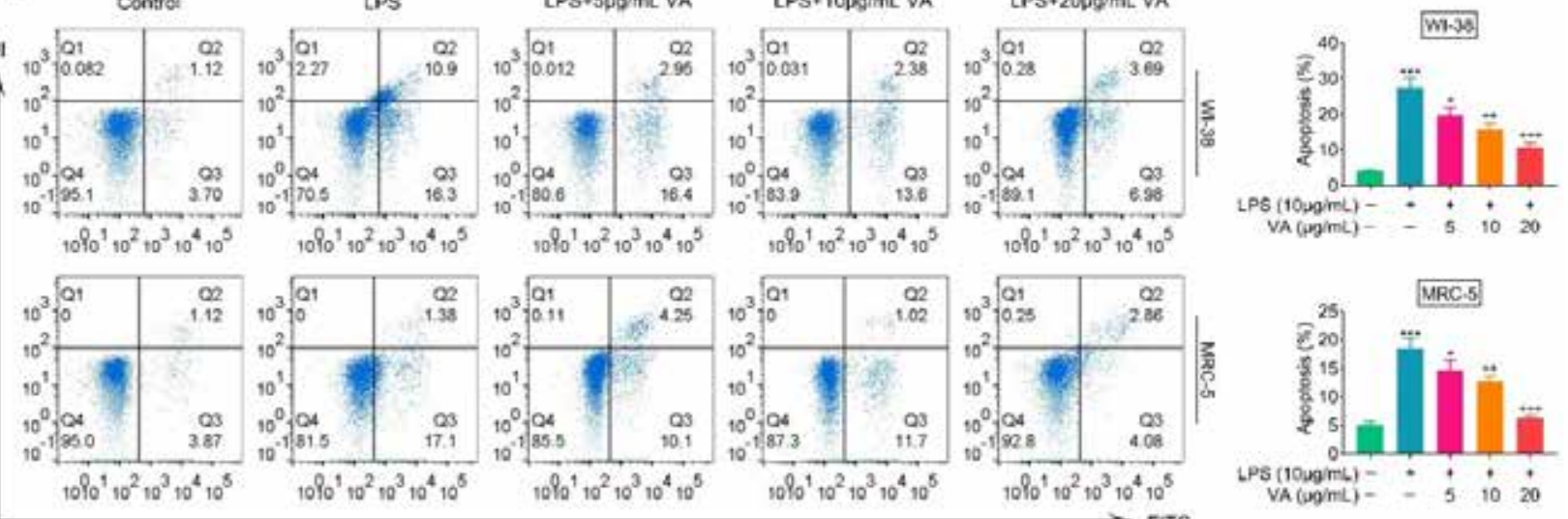

(E)
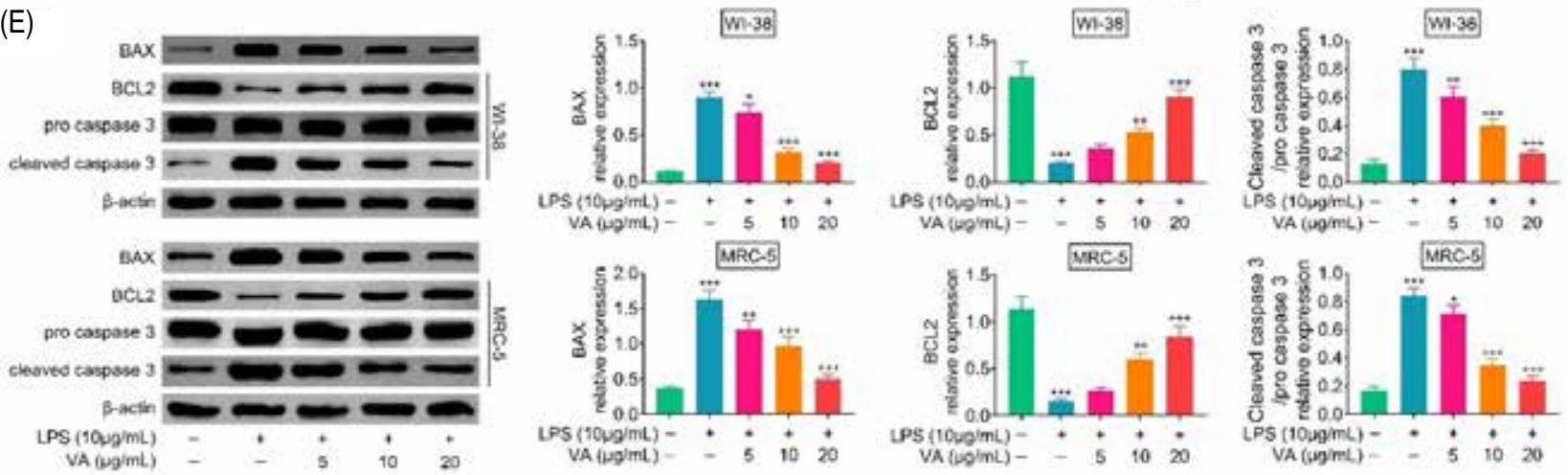

Figure 1. Vanillic acid suppressed cell apoptosis of lipopolysaccharides-treated human lung fibroblasts. (A) Incubation with lipopolysaccharides (more than $2 \mu \mathrm{g} / \mathrm{mL}$ ) reduced cell viabilities of WI-38 and MRC-5. (B) Vanillic acid $(40 \mu \mathrm{g} / \mathrm{mL}$ ) reduced cell viabilities of WI-38 and MRC-5, while vanillic acid below $20 \mu \mathrm{g} / \mathrm{mL}$ demonstrated no significant effect on the cell viabilities of WI-38 and MRC-5. (C) Vanillic acid enhanced cell viabilities of lipopolysaccharides-treated WI-38 and MRC-5 in a dosagedependent manner. (D) Vanillic acid treatment suppressed cell apoptosis of lipopolysaccharides-treated WI-38 and MRC-5 in a dosage-dependent manner. (E) Vanillic acid treatment decreased protein expression of $B A X$ while increasing $B C L 2$ and cleaved caspase 3 in lipopolysaccharides-treated WI-38 and MRC-5. ${ }^{*} P<0.05,{ }^{* *} P<0.01,{ }^{* *} P<0.001$.

(Figure 4A), expressions of p-JNK and p-ERK were increased in lipopolysaccharides-treated WI-38 and MRC-5 (Figure 4A). Moreover, vanillic acid decreased p-JNK and p-ERK expressions in a dosage-dependent manner (Figure 4A). Similarly, vanillic acid attenuated lipopolysaccharides-induced down-regulation of $\mathrm{I} \kappa \mathrm{B} \alpha$, and up-regulation of p-p65 and p-IкB $\alpha$ in WI-38 and MRC-5 (Figure 4B), revealing that vanillic acid inactivated MAPK and NF- $\mathrm{KB}$ pathways in lipopolysaccharides-treated human lung fibroblasts.

\section{Discussion}

Pneumonia is known as an inflammatory lung manifestation stimulated by bacterial or viral infections (Mizgerd, 
(A)
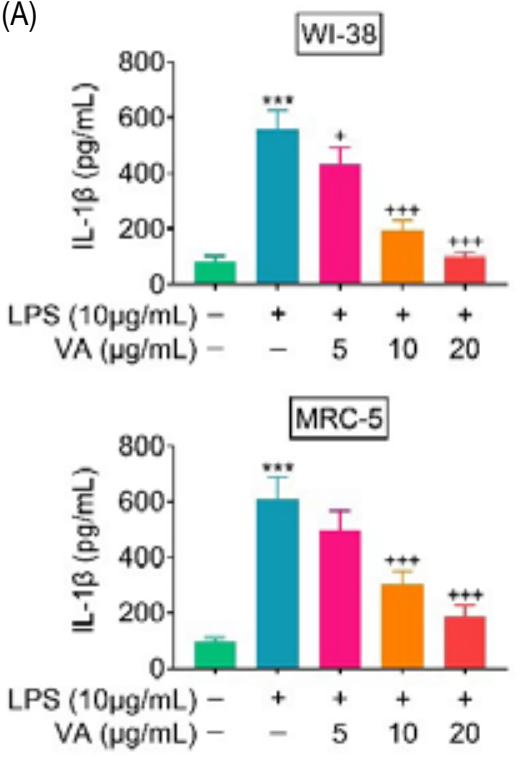

(B)
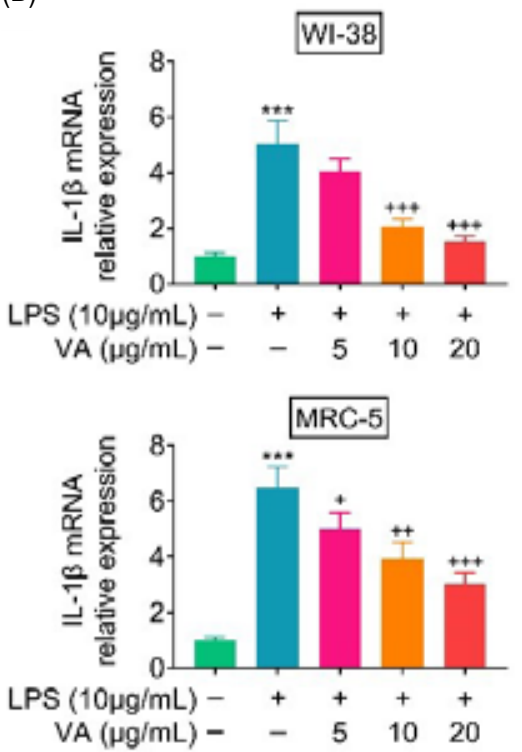
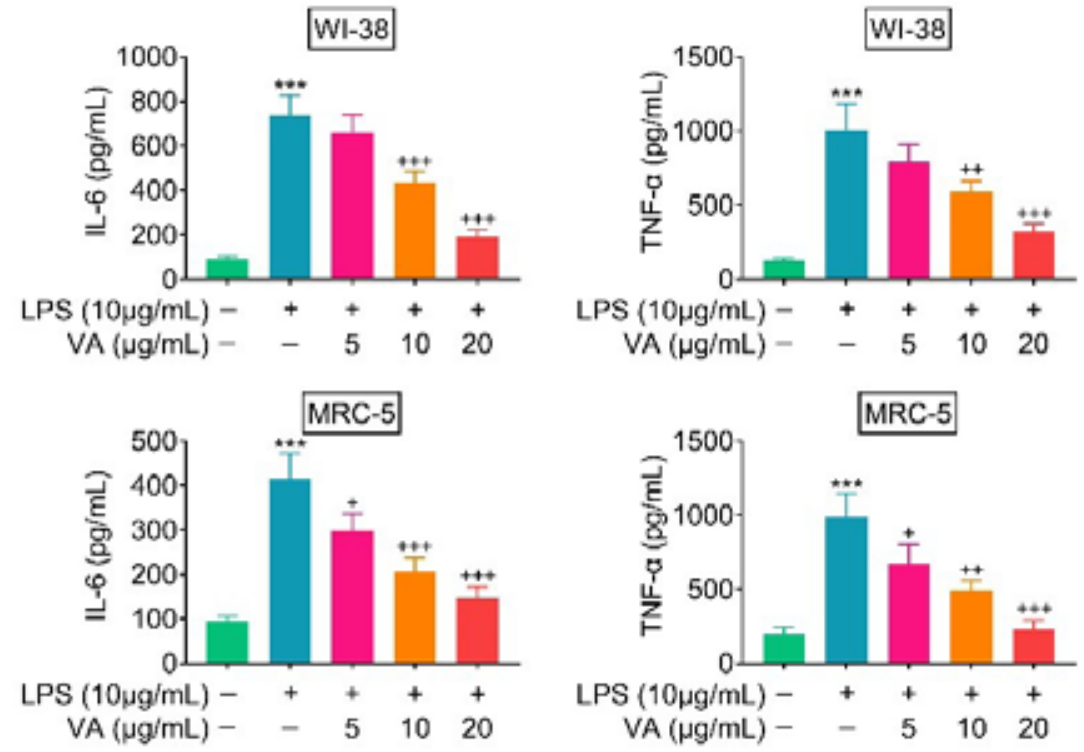

Figure 2. Vanillic acid suppressed inflammation of lipopolysaccharides-treated human lung fibroblasts. (A) Vanillic acid treatment reduced protein expression of TNF- $\alpha$, IL- 6 , and IL- $1 \beta$ in lipopolysaccharides-treated WI- 38 and MRC- 5 in a dosagedependent manner. (B) Vanillic acid treatment reduced mRNA expression of TNF- $\alpha$, IL-6, and IL-1 $\beta$ in lipopolysaccharides-treated WI-38 and MRC-5 in a dosage-dependent manner. ${ }^{+} P<0.05,{ }^{++} P<0.01,{ }^{+++}{ }^{* * *} P<0.001$.

2018). Dietary fruit and vegetation endowed with antiinflammatory and antioxidant properties have been used for the prevention of pulmonary diseases (Scoditti et al., 2019), including pneumonia (Simopoulos, 2021). The anti-inflammatory, antimicrobial, antioxidant, and neuroprotective effects of vanillic acid have been studied widely (Ullah et al., 2020). The present study established that vanillic acid suppressed endoplasmic reticulum stress-induced inflammation in human lung fibroblasts, thus providing potential strategy for the prevention of pneumonia.
During the development of pneumonia, lung inflammation was promoted and the inflammatory cytokines were released to induce cellular damages (Tang et al., 2020). Lipopolysaccharides are widely used to induce inflammation in human lung fibroblasts, thus mimicking cell model of pneumonia (Tang et al., 2020). Alleviation of lipopolysaccharides-induced inflammation contributed to the prevention of pneumonia (Quan et al., 2018). Results in this study established that treatment with lipopolysaccharides reduced cell viability of human lung fibroblasts, promoted cell apoptosis, and 

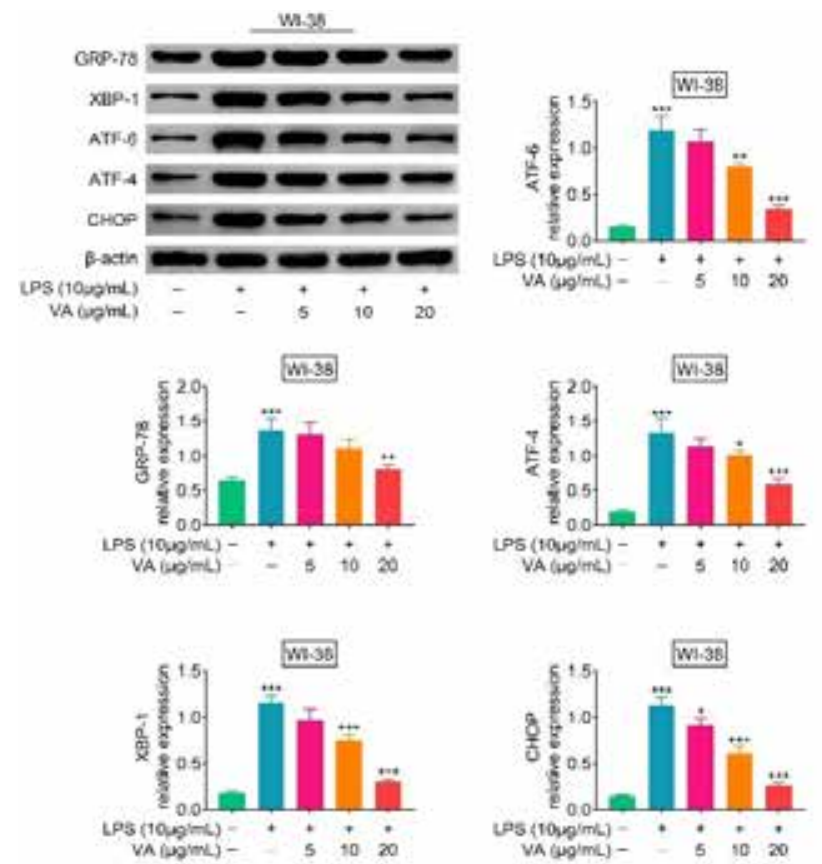
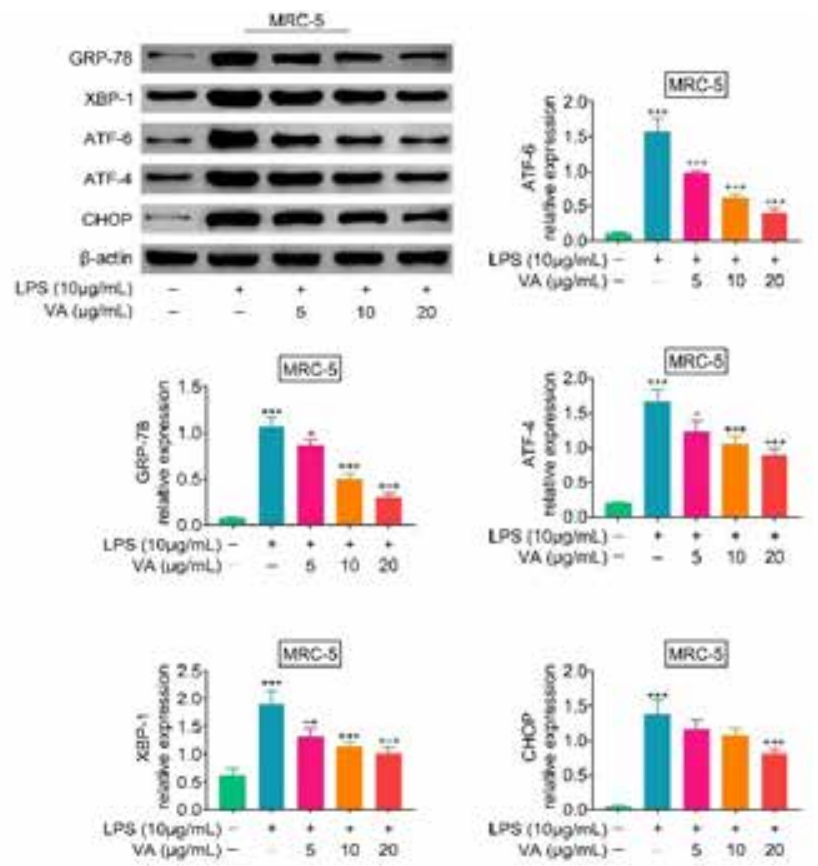

Figure 3. Vanillic acid suppressed endoplasmic reticulum stress of lipopolysaccharides-treated human lung fibroblasts. Vanillic acid treatment reduced the expression of GRP-78, XBP-1, ATF-6, ATF-4, and CHOP in lipopolysaccharides-treated WI-38 and MRC-5 in a dosage-dependent manner. ${ }^{+} P<0.05,{ }^{++} P<0.01,{ }^{+++,}{ }^{* * *} P<0.001$.

induced the secretion of inflammatory factors, including TNF- $\alpha$, IL-6, and IL-1 $\beta$. However, vanillic acid exerted anti-apoptotic and anti-inflammatory effects on lipopolysaccharides-treated human lung fibroblasts through up-regulation of $B C L 2$, and down-regulation of $B A X$, cleaved caspase 3, TNF- $\alpha$, IL-6, and IL-1 $\beta$.

Endoplasmic reticulum stress has long been considered to be a critical regulator of systemic inflammatory response (Zhang and Kaufman, 2008). Lipopolysaccharides are indicated to up-regulate protein expressions of ATF6, XBP1, GRP78, and CHOP to induce endoplasmic reticulum stress and promote the activation of inflammatory response in acute lung injury model (Zeng et al., 2017). Suppression of endoplasmic reticulum stress reduced lipopolysaccharides-induced lung inflammation (Kim et al., 2013). This study also found that endoplasmic reticulum stress in human lung fibroblasts was promoted by lipopolysaccharides treatment with up-regulation of GRP-78, XBP-1, ATF-6, ATF-4, and CHOP. However, vanillic acid suppressed lipopolysaccharides-induced endoplasmic reticulum stress in human lung fibroblasts, thus alleviating lung inflammation.

A previous study has established that NF- $\kappa B$ and MAPK signaling is a common pro-inflammatory pathway to induce the expression of pro-inflammatory cytokines chemokines, and adhesion molecules (Xiao et al., 2020). Moreover, NF-KB and MAPK were activated by lipopolysaccharides stimulation of lipopolysaccharides to regulate expression of pro-inflammatory cytokines (Nguyen et al., 2020). Inactivation of NF-KB and MAPK pathways contributed to the amelioration of lipopolysaccharides-induced lung inflammation (Chi et al., 2012) and endoplasmic reticulum stress (Huang et al., 2020). Vanillic acid has been reported to suppress lipopolysaccharides-stimulated activation of NF- $\mathrm{KB}$ to reduce inflammatory response (Calixto-Campos, 2015; Kim et al., 2011). In addition, vanillic acid also inactivated lipopolysaccharides-stimulated MAPK signaling to alleviate neurotoxicity (Ullah et al., 2020), and attenuated IL-1 $\beta$-stimulated cartilage degeneration by suppressing MAPK and PI3K/AKT/NF-KB pathways (Huang et al., 2019). Here, lipopolysaccharides stimulated activation of MAPK and NF- $\mathrm{KB}$ pathways in human lung fibroblasts with down-regulation of NF- $\kappa B$ inhibitor, I $\kappa B \alpha$, and up-regulation of p-p65, p-I $\mathrm{kB} \alpha$, p-JNK, and p-ERK. However, MAPK and NF-kB pathways in lipopolysaccharides-stimulated human lung fibroblasts were suppressed by vanillic acid through reversed effects on IKB $\alpha, \mathrm{p}-\mathrm{p} 65$, $\mathrm{p}$-IKB $\alpha, \mathrm{p}$-JNK, and $\mathrm{p}$-ERK expressions.

\section{Conclusion}

Vanillic acid exerted anti-apoptotic, anti-inflammatory, and anti-endoplasmic reticulum stress effects against lipopolysaccharides-stimulated human lung fibroblasts through inactivation of MAPK and NF- $\mathrm{KB}$ 
(A)
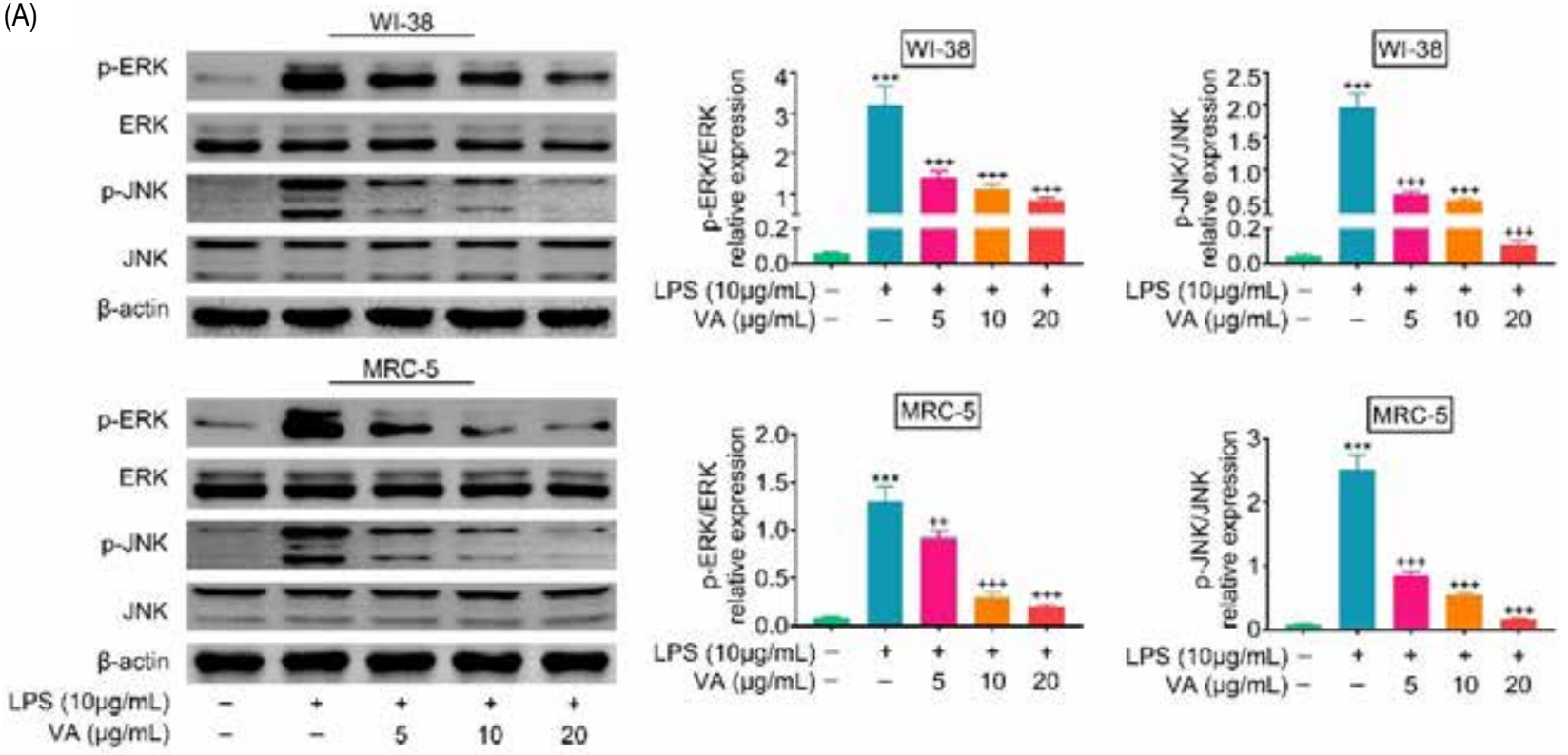

(B)
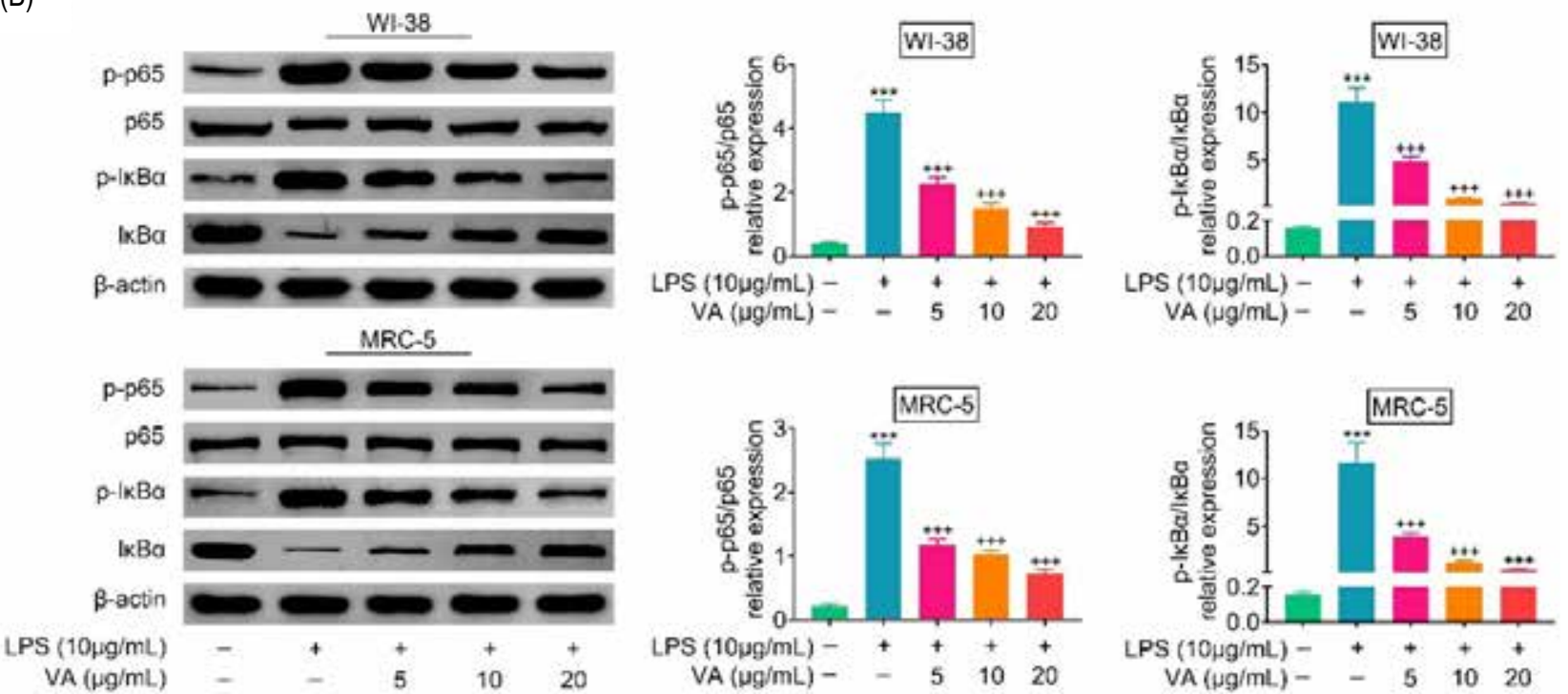

Figure 4. Vanillic acid suppressed activation of MAPK and NF-kB pathways. (A) Vanillic acid decreased $p$-JNK and p-ERK expression in a dosage-dependent manner. (B) Vanillic acid attenuated lipopolysaccharides-induced down-regulation of $I_{K} B \alpha$, and up-regulation of $\mathrm{p}-\mathrm{p} 65$ and $\mathrm{p}-\mathrm{I} \mathrm{KB} \alpha$ in WI-38 and MRC-5. ${ }^{+++, * * *} P<0.001$.

pathways. Therefore, vanillic acid might be developed into a promising therapeutic factor for preventing pneumonia. However, the effect of vanillic acid on the oxidative stress of lipopolysaccharides-stimulated human lung fibroblasts, as well as the in vivo effect on animal model of pneumonia, must be investigated in the future research.

\section{Competing interests}

The authors state that there are no conflicts of interest to disclose.

\section{Author Contribution}

Jihua Zhao and Yao Yang designed the experiments and carried them out. Jihua Zhao analyzed and interpreted the data, Yao Yang prepared the manuscript with contributions from all co-authors.

\section{Reference}

Amin, F.U., Shah, S.A. and Kim, M.O., 2017. Vanillic acid attenuates A $\beta 1-42$-induced oxidative stress and cognitive impairment in mice. Scientific Reports 7: 40753. https://doi.org/10.1038/srep40753 
Bai, F., Fang, L., Hu, H., Yang, Y., Feng, X. and Sun, D., 2019. Vanillic acid mitigates the ovalbumin (OVA)-induced asthma in rat model through prevention of airway inflammation. Bioscience, Biotechnology, and Biochemistry 83: 531-537. https://doi.org/1 0.1080/09168451.2018.1543015

Calixto-Campos, C.S., 2015. Vanillic acid inhibits inflammatory pain by inhibiting neutrophil recruitment, oxidative stress, cytokine production, and NFKB activation in mice. Journal of Natural Products 78: 1799-1808. https://doi.org/10.1021/acs. jnatprod.5b00246

Chi, G., Wei, M., Xie, X., Soromou, L.W., Liu, F. and Zhao, S., 2012. Suppression of MAPK and NF- $\mathrm{KB}$ pathways by limonene contributes to attenuation of lipopolysaccharide-induced inflammatory responses in acute lung injury. Inflammation 36(2): 501-511. https://doi.org/10.1007/s10753-012-9571-1

Dassner, M.A., Nicolau, P.D. and Girotto, E.J., 2017. Management of pneumonia in the pediatric critical care unit: an area for antimicrobial stewardship. Current Pediatric Reviews 13: 49-66. https://doi.org/http://dx.doi.org/10.2174/157339631266616120 5102221

Huang, C.-Y., Deng, J.-S., Huang, W.-C., Jiang, W.-P. and Huang, G.-J., 2020. Attenuation of lipopolysaccharide-induced acute lung injury by hispolon in mice, through regulating the TLR4/PI3K/ Akt/mTOR and Keap1/Nrf2/HO-1 pathways, and suppressing oxidative stress-mediated ER stress-induced apoptosis and autophagy. Nutrients 12: 1742.

Huang, X., Xi, Y., Mao, Z., Chu, X., Zhang, R., Ma, X., Ni, B., Cheng, H. and You, H., 2019. Vanillic acid attenuates cartilage degeneration by regulating the MAPK and PI3K/AKT/NF- $\mathrm{BB}$ pathways. European Journal of Pharmacology 859: 172481. https://doi.org/https://doi.org/10.1016/j.ejphar.2019.1724.81

Hur, I., Ozkan, S., Halici, A., Abatay, K., Usul, E., Cetin, E. and Aydin, F.N., 2020. Role of plasma presepsin, procalcitonin and $\mathrm{C}$-reactive protein levels in determining the severity and mortality of community-acquired pneumonia in the emergency department. Signa Vitae 16: 61-68.

Ji, G., Sun, R., Hu, H., Xu, F., Yu, X., Veeraraghavan, V., Krishna Mohan, S. and Chi, X., 2020. Vannilic acid ameliorates hyperglycemia-induced oxidative stress and inflammation in streptozotocin-induced diabetic rats. Journal of King Saud University - Science 32(7): 2905-2911. https://doi.org/10.1016/j.jksus.2020.04.010

Kim, H.J., Jeong, J.S., Kim, S.R., Park, S.Y., Chae, H.J. and Lee, Y.C., 2013. Inhibition of endoplasmic reticulum stress alleviates lipopolysaccharide-induced lung inflammation through modulation

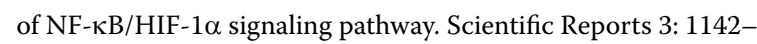
1142. https://doi.org/10.1038/srep01142

Kim, M.-C., Kim, S.-J., Kim, D.-S., Jeon, Y.-D., Park, S.J., Lee, H.S., Um, J.-Y. and Hong, S.-H., 2011. Vanillic acid inhibits inflammatory mediators by suppressing NF- $\mathrm{KB}$ in lipopolysaccharide-stimulated mouse peritoneal macrophages. Immunopharmacology and Immunotoxicology 33: 525-532. https://doi.org/10.3109/08923973.2010.547500

Köseler, A., Sabirli, R., Gören, T., Türkçüer, I. and Kurt, Ö., 2020. Endoplasmic reticulum stress markers in SARS-COV-2 infection and pneumonia: case-control study. In Vivo (Athens, Greece) 34: 1645-1650. https://doi.org/10.21873/invivo.11956
Marangu, D. and Zar, H.J., 2019. Childhood pneumonia in low-andmiddle-income countries: an update. Paediatric Respiratory Reviews 32: 3-9. https://doi.org/10.1016/j.prrv.2019.06.001

Mizgerd, J.P., 2018. Inflammation and pneumonia: why are some more susceptible than others? Clinics in Chest Medicine 39: 669-676. https://doi.org/10.1016/j.ccm.2018.07.002

Müller-Redetzky, H., Lienau, J., Suttorp, N. and Witzenrath, M., 2015. Therapeutic strategies in pneumonia: going beyond antibiotics. European Respiratory Review 24: 516. https://doi. org/10.1183/16000617.0034-2015

Nguyen, T., Binh, T., Kusunoki, R., Pham, T., Nguyen, H.Y., Tuân, N., Kanaori, K. and Kamei, K., 2020. Effects of Launaea sarmentosa extract on lipopolysaccharide-induced inflammation via suppression of NF- $\mathrm{KB} / \mathrm{MAPK}$ signaling and Nrf2 activation. Nutrients 12(9): 2586. https://doi.org/10.3390/ nu12092586

Quan, B., Zhang, H. and Xue, R., 2019. miR-141 alleviates LPSinduced inflammation injury in WI-38 fibroblasts by up-regulation of NOX2. Life Sciences 216: 271-278. https://doi. org/10.1016/j.lfs.2018.11.056

Scoditti, E., Massaro, M., Garbarino, S. and Toraldo, D.M., 2019. Role of diet in chronic obstructive pulmonary disease prevention and treatment. Nutrients 11: 1357. https://doi.org/10.3390/ nu11061357

Seki, H., Fukunaga, K., Arita, M., Arai, H., Nakanishi, H., Taguchi, R., Miyasho, T., Takamiya, R., Asano, K., Ishizaka, A., Takeda, J. and Levy, B.D., 2010. The anti-inflammatory and proresolving mediator resolvin E1 protects mice from bacterial pneumonia and acute lung injury. Journal of Immunology (Baltimore, MD) 184: 836-843. https://doi.org/10.4049/ jimmunol.0901809

Siddiqui, S., Kamal, A., Khan, F., Jamali, K.S. and Saify, Z.S., 2019. Gallic and vanillic acid suppress inflammation and promote myelination in an in vitro mouse model of neurodegeneration. Molecular Biology Reports 46: 997-1011. https://doi. org/10.1007/s11033-018-4557-1

Simopoulos, A.P., 2021. Genetic variation, diet, inflammation, and the risk for COVID-19. Lifestyle Genomics 14: 37-42. https:// doi.org/10.1159/000513886

Tang, X., Wang, T., Qiu, C., Zheng, F., Xu, J. and Zhong, B., 2020. Long non-coding RNA (lncRNA) CRNDE regulated lipopolysaccharides (LPS)-induced MRC-5 inflammation injury through targeting MiR-141. Medical Science Monitor: International Medical Journal of Experimental and Clinical Research 26: e920928-e920928. https://doi.org/10.12659/ MSM.920928

Ullah, R., Ikram, M., Park, T.J., Ahmad, R., Saeed, K., Alam, S.I., Rehman, I.U., Khan, A., Khan, I., Jo, M.G. and Kim, M.O., 2020. Vanillic acid, a bioactive phenolic compound, counteracts LPSinduced neurotoxicity by regulating c-Jun $\mathrm{N}$-terminal kinase in mouse brain. International Journal of Molecular Sciences 22: 361. https://doi.org/10.3390/ijms22010361

Xiao, K., Liu, C., Tu, Z., Xu, Q., Chen, S., Zhang, Y., Wang, X., Zhang, J., Hu, C.-A.A. and Liu, Y., 2020. Activation of the $\mathrm{NF}-\mathrm{\kappa B}$ and MAPK signaling pathways contributes to the inflammatory responses, but not cell injury in IPEC-1 cells 
challenged with hydrogen peroxide. Oxidative Medicine and Cellular Longevity 2020: 5803639. https://doi.org/10.1155/ 2020/5803639

Zeng, M., Sang, W., Chen, S., Chen, R., Zhang, H., Xue, F., Li, Z., Liu, Y., Gong, Y., Zhang, H. and Kong, X., 2017. 4-PBA inhibits LPS-induced inflammation through regulating ER stress and autophagy in acute lung injury models. Toxicology Letters 271: 26-37. https://doi.org/https://doi.org/10.1016/j. toxlet.2017.02.023

Zhang, K. and Kaufman, R.J., 2008. From endoplasmic-reticulum stress to the inflammatory response. Nature 454: 455-462. https://doi.org/10.1038/nature07203 